\title{
Research on the Image De-noising Algorithm Based on Wiener Filtering and Edge Modeling
}

\author{
Chuan Tian ${ }^{1, ~ a, ~ X i n ~} \mathrm{Li}^{2, \mathrm{~b}}$ and Peng Wang ${ }^{3, \mathrm{c}}$ \\ ${ }^{1}$ College of Software, Tsinghua University, Beijing 100081, China \\ ${ }^{2}$ Information Department Academy of Armored Force Engineering Beijing, 100072, China \\ ${ }^{3}$ Central Banks of Qingdao city, Qingdao 266071, China \\ aTianchuan_zbj@126.com, ${ }^{\mathrm{b}}$ Lixin_522088@126.com, ${ }^{\mathrm{C}}$ pengw@yeah.net
}

Keywords: De-noising; Wiener filtering; Edge modeling; Mathematical analysis; Algorithm

\begin{abstract}
In this paper, we conduct research on the image de-noising algorithm based on the Wiener filtering and the edge modeling. Image restoration is a norm and the noise pollution observation to estimate the original image in the image, the purpose is to restore the decay and loss of high frequency components. In real applications, the image degradation process is very complicated, it is difficult to describe with accurate mathematical model and therefore the applicable scope of the core traditional image restoration technology is limited. Under this background, we propose the new perspectives of the issues that will be meaningful and necessary.
\end{abstract}

\section{Introduction}

The traditional linear or nonlinear filtering is indiscriminate convolution operation for the image as a whole, this is often on the noise section equally enhancement processing, the edge of the object in the remote sensing images, especially in the remote sensing images or grayscale change relatively quickly becomes smooth, harmful to interpretation work.

Linear filter, such as minimum mean square filtering, average filtering for in the image de-noising after make the negative impact of the edge of the image blur, namely median filter can eliminate the noise in the image, as well as protect the edges of the image and contour. According to the literature review, we could summarize the filtering methodologies into the listed aspects. (1) Multistage median filtering. In multistage median filtering algorithm, after two median filtering, and increased with the center pixel edge correlation of pixels of the weight, reduce the weight of the impulse noise and its neighboring pixels. (2) Switching median filter. In the concentrated distribution of the noise in the binary image, the distribution of noise point lattice will be easier to get than from the original image. For grayscale distribution diversity of natural images, we adopt the method of the statistics to get the threshold value as these thresholds will play an important role to control switch the median filtering. (3) Extremum median filter. The method introduced core preliminary judgment operator to make the image area more detailed classification, so as to achieve the suppress noise and protect the details of the good effect, and has a better adaptability to noise [1-3].

Image restoration is a norm and noise pollution observation to estimate the original image in the image, the purpose is to restore the decay and loss of high frequency components. Deconvolution is the norm of the inverse process, often sick, generally using regularization method, makes the solution of the inverse problem of identifying and stability, thus effectively restore the original image. If the LTI wiener filter is used to estimate restoration, noise reduction of smooth all the signal component at the same time while make the recovery image edge contour fuzzy. Wavelet transform has the good time-frequency local features as can be very effective in analyzing non-stationary signals: the rational use of the wavelet threshold estimation that can effectively restrain noise, restore the image.

For the optimization of the filtering algorithms, we summarize the advantages of the traditional approaches. (1) The classic wiener filtering. Classic wiener filter is a linear filter, used to extract useful signal from noise. The classic wiener filtering method is designed according to the minimum 
mean square error criterion as is mainly composed of before all the general measurements and current measurements to estimate the current value of a signal filtering. (2) Average filtering. This method makes the noise intensity is abate, the original gray levels of the pixels more prominent can suppress noise and has good inhibition effect on the Gaussian noise. But the average neighborhood will make images appear blurred border. (3) Median filtering. Median filtering can remove noise particles, can protect the edges of the image, therefore, in the space of impulse noise is not too big can achieve very good effect of noise. In the Fig. 1, we demonstrate the sample [4-5].
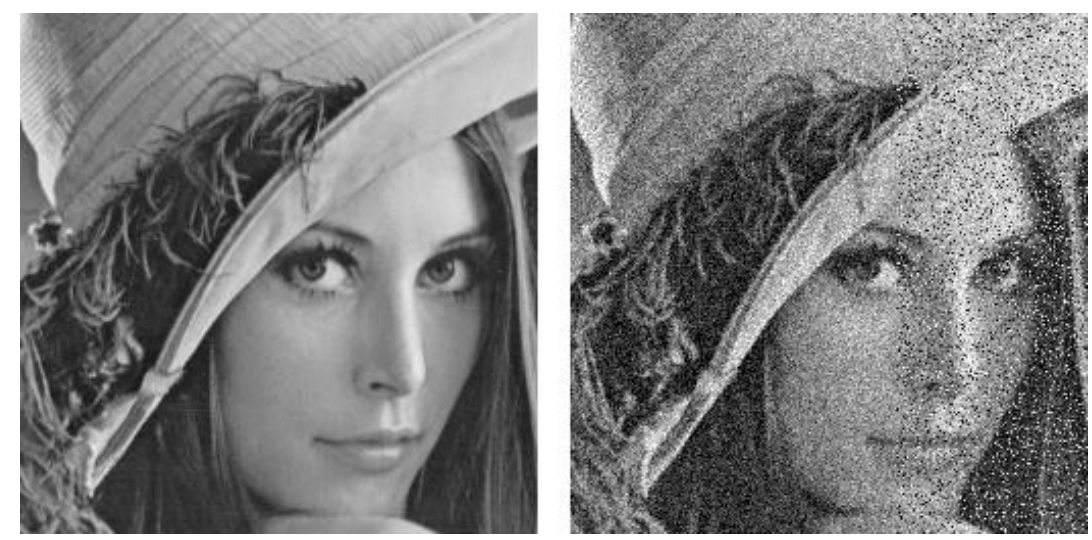

Figure 1. The Visualized Illustration of the Image De-noising Performance

In this paper, we conduct research on the image de-noising algorithm based on Wiener filtering and the edge modeling. In the later sections, we will discuss the issues in detail.

\section{Our Proposed Methodology and Algorithm}

The Image Gradient. Many researchers found that the human eye for image edge and basic texture information is the most sensitive and important, that is, the structure of the edge and basic texture information is likely to be the most important part of the image structure information. The third part, as the most important of SSIM structure information is essentially to calculate only the original image sub-block and drop the image pixel values of the correlation coefficient between sub-block, as cannot well reflect the image edge and texture information, so for the blurred image, because it can't measure the original image and a drop in quality, and the image edge structure information of the differences between the SSIM model showed poor objective evaluation results.

In real applications, the image degradation process is very complicated, it is difficult to describe with accurate mathematical model and therefore the applicable scope of the core traditional image restoration technology is limited. Blind image restoration algorithm can, in contrast, primary image degradation under unknown kernel function, direct use of the general degraded images to estimate the degradation of kernel function and restore clear images, so this kind of basic algorithm has stronger practicability. Inflation and corrosion can be used for edge detection, but does not apply to contain the noise of the image processing; Opened and closed operation to noise effect is good. Therefore, based on the theory of morphological gradient morphology double gradient operator is proposed.

Some scholars from the perspective of parametric spline said, under a given function fitting error of the order, by minimizing the fitting error constant got some optimal interpolation basis function, the basic model is as follows.

$$
f(t)=\sum_{n} C_{n} \lambda\left(\frac{t}{T}-n\right)
$$

Statistical method is, in fact, the problem of image interpolation is transformed into a functional minimization problem with constraints, this is equivalent to the given probability density function of 
the original signal and observation data, the image interpolation problem is transformed into the best estimation of original signal that could be expressed as follows.

$$
\operatorname{MSSIM}(X, Y)=\frac{1}{M} \sum_{j=1}^{M} \operatorname{SSIM}\left(x_{j}, y_{j}\right)
$$

SSIM method based on image structure information, this paper presents the innovation idea, and thus has obtained the good effect of judgment, but with the deepening of the research, we found that this method has some defects. On second order continuous differentiable function interpolation, stay around interpolation point's value and its value than closer to a flat point, that is to say, when using its about two interpolation, the smaller the derivative of the corresponding weights of the greater.

$$
A m p_{i, j}=\left|d x_{i, j}\right|+\left|d y_{i, j}\right|
$$

Accordingly, each pixel is defined as the gradient direction as follows.

$$
A n g_{i, j}=\frac{180^{\circ}}{\pi} \times \arctan \left(\frac{d y_{i, j}}{d x_{i, j}}\right)
$$

Level set curve in the process of evolution, in order to keep as the symbolic distance function, in every step or every few steps after the evolution of the initial level set initialized. To initialize level set will lead to the following results: 1 . The level set to initialize level set theory and the inconsistencies between its implementation; 2. After the re-initialization of the level set may lead to zero level set gravely deviates from the original position; 3 . In the process of evolution, when to initialize and how to implement the re-initialization is also a very difficult problem. What is more important to initialize level set will increase a lot of calculation work, so as to make the evolution speed is very slow.

$$
p_{\text {enhanced }}(u)=\iint_{\theta} \frac{1}{2}(|\nabla u|-1)^{2} d x d y
$$

The Wiener Filtering. Classic wiener filter is a kind of based on the core local variance automatic adjustment of adaptive filter, the effect of filter than linear filter has better selectivity, can intact image edges and details. But the classical adaptive wiener filtering is in with the mean and variance of fixed template selected adaptively determine the output value as is just a simple adaptively adjust the input value and output value. In the classic wiener filtering, need to estimate the image noise variance this is hard to do in the practical application [6-7].

To get the optimum wiener filter, we must know the original $\mathrm{x}$ and no noise image noise variance of wavelet coefficients. The latter use based on the best measure of noise image y wavelet coefficients of the median filter can get a good approximation to estimate, while the former is hard to come by. In order to get the optimal estimation of $\mathrm{x}$, $\mathrm{y}$ with the noise image in the first wavelet domain threshold de-noising image estimation model $\mathrm{x} 1$, and then noise image and estimation model $\mathrm{x} 1, \mathrm{y}$ in the second orthogonal transform, the wavelet domain using the wiener filter to get the original image of the final estimate. Considering the first function of wavelet domain estimation model $\mathrm{x} 1$, with no noise model mismatch, continued in the first wavelet domain wiener filtering will not improve this situation, so the wiener filtering option in the wavelet domain as the follows.

$$
\theta(i, j)=H_{w}(i, j) w_{2}(i, j)
$$

After calculating variance of all the pixels, the statistical average values of variance of image, first for a single pixel, using the method, shown in the image of each pixel in the mean and the variance are compared under different templates, take out the window of the minimum average mean when used as the pixels to the final processing window of wiener filtering. Use the following modified formula for pixel processing, the output results.

$$
r(i, j)=\delta+(1-q+\Delta) \otimes(s(i, j)-\lambda)
$$

In the treatment of order 3, 5, 7 and 9 order data, not every template data calculated separately, but the application of the calculation data accumulation of three order windows to the other order. In this way, the spatial location nearby and has similar grey value point will be similar to that of output pulse, and space position and gray value differences far point get different output pulse, and by using the 
properties of the image itself to analyze general image, realized by image coefficient to neurons firing frequency nonlinear mapping that could be revised as the formula 8 .

$$
y \rightarrow Y \quad H \rightarrow h
$$

Relative to some other wavelet threshold methods, wavelet domain wiener filter with two wavelet transform domain processing reduces the estimated image signal model and the real image signal model mismatch degree, thus reducing the resulting error, and achieve the optimal estimate. In the following Fig. 2, we demonstrate the corresponding features.

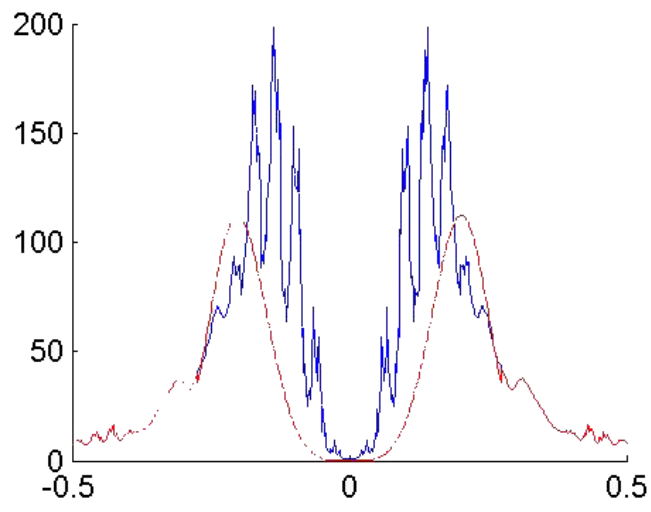

Figure 2. The Wiener Filtering Illustration

The Edge Modeling. Edge character is the most useful information in image detection. In image de-noising, image edge character as far as possible, is to focus on the problems in image de-noising. The edge detection procedures could be separated into the listed steps.

- Edge detection algorithm is mainly based on the image of the first and second order derivative, but derivative calculation is sensitive to noise, so you must use the filter to improve the edge detector is related to the noise performance.

- At various points in the foundation is to determine the image edge enhancement neighborhood intensity values. Enhancement algorithm neighborhood strength value that can be significant change points are highlighted [8].

- There are many points in the image of the gradient amplitude is large, and these points in the specific application areas are not edge, should use some method to determine the edge points are those. The simplest edge detection criterion is gradient amplitude threshold criterion.

- If applications require edge position is determined, the edge position can be estimated in the sub pixel resolution, on the edge of the bearing can be estimated.

So far, in the international conference on journals have been published in a lot of the papers about color image edge detection, most of these methods are tried to gray image edge detection method is applied to general color images edge detection. The algorithm firstly by the color of luminance and chrominance information separately for different processing, and gives different weights, and then, to quantify the difference between the color finally gray image edge detection template that is used to calculate the gradient image, for the image edge. The color difference of color distance calculation, as weight calculation and the distance of synthesis of three parts. 


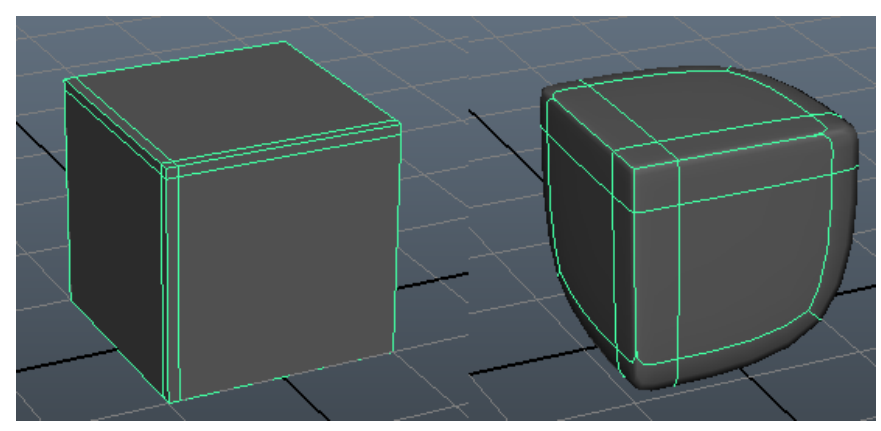

Figure 3. The Demonstration of the Edge Modeling Technique

Gradient corresponding to the first derivative, gradient operator is the first derivative operator. On the edge of gray value transition is more acute, and the image noise is small, the effect of the gradient operator work better, and to exert the operation direction as the follows [9].

$$
\nabla f(x, y)=\left[G_{x}, G_{y}\right]^{T}=\left[\frac{\partial f}{\partial x} \frac{\partial f}{\partial y}\right]^{T}
$$

In the application of the template, it is necessary to remove the dc component, even if the template matrix of each the element is zero. This is because when the image and template convolution, core component is not equal to zero, that will lead to the changes in the number of passing zero, thus makes edge detection position is not accurate. The enhance parameter is shown as follows.

$$
\vartheta(x, y)=\arctan \left(\left[\frac{\partial f}{\partial x}\right] /\left[\frac{\partial}{\partial y}\right]\right)
$$

\section{Conclusion and Summary}

In this paper, we conduct research on the image de-noising algorithm based on Wiener filtering and the edge modeling. Under the same mis-judgement, based on binary wavelet transform de-noising can improve the image reconstruction effects. On the basis of this thought, in this paper, from the research domain, and noise in the wavelet series of relevant features of binary wavelet transform domain, this paper proposes a de-noising method to existing wavelet series of binary wavelet domain de-noising method. Under this background, we propose the Wiener filtering and edge modeling based method that will enhance the traditional approaches.

\section{References}

[1] Gecui, G. O. N. G., M. A. Lei, and L. I. U. Jianping. "Research on the Wavelet Transform Based Image De-noising Algorithm for Adaptive Fuzzy Threshold." Journal of Convergence Information Technology 7.11 (2012).

[2] Xin, Qiao. "The principle curvature-driven diffusion model for image de-noising." (2013).

[3] Ray, Priyadip, A. K. Maitra, and Arijit Basuray. "A new threshold function for de-noising partial discharge signal based on wavelet transform." Signal Processing Image Processing \& Pattern Recognition (ICSIPR), 2013 International Conference on. IEEE, 2013.

[4] Sulaiman, Siti Noraini, et al. "De-noising of noisy MRI brain image using the switching-based clustering algorithm." Control System, Computing and Engineering (ICCSCE), 2014 IEEE International Conference on. IEEE, 2014.

[5] Hussain, Rashid, Abdul Rehman Memon. "Selecting best mother wavelets for curvelet transform based image de-noising." JOURNAL OF ENGINEERING RESEARCH 1.1 (2013): 201-212. 
[6] Chowdhury, M. Mozammel Hoque. "A Robust De-Noising Model for Image Enhancement with Adaptive Median Filtering." American Journal of Modeling and Optimization 2.3 (2014): 69-72.

[7] Lin, Zhen Xian. "A Improved Algorithm of Wavelet Image De-Noising Based on Threshold Function." Advanced Materials Research. Vol. 756. 2013.

[8] Wang, YuLing, Ming Li, and Li Li. "An image denoising algorithm based on clustering and median filtering." Sixth International Conference on Graphic and Image Processing (ICGIP 2014). International Society for Optics and Photonics, 2015.

[9] Shettar, Jyoti, Ekta Maini, and S. Shreelakshmi. "Image Sharpening \& De-Noising Using An Adaptive Bilateral Filter." International Journal of Innovative Research and Development 2.11 (2013). 